Nervenarzt 2017 $\cdot 88: 343-344$

DOI 10.1007/s00115-017-0303-5

Online publiziert: 13. März 2017

(c) Springer Medizin Verlag GmbH 2017

CrossMark

\title{
D. Berg
}

Klinik für Neurologie, Christian-Albrechts-Universität zu Kiel, Kiel, Deutschland

\section{Parkinson - 200 Jahre nach Erstbeschreibung der Schüttellähmung}

[2] und beginnt lange (nach heutigem Wissen Jahre bis Jahrzehnte), bevor der Betroffene - mit Worten von J. Parkinson „Leidende“ („sufferer“) - erste Symptome wahrnimmt.

Wo stehen wir 200 Jahre später?

Intensive, ernsthafte Forschung von Generationen von Wissenschaftlern und ein immenser methodischer und technischer Fortschritt weisen heute auf eine multifaktorielle Genese der ParkinsonErkrankung hin, wobei auch bei der den größten Anteil ausmachenden idiopathischen Form eine genetische Mitbeteiligung wahrscheinlich ist (siehe Artikel von Lill und Klein in diesem Heft). Weder für das Verständnis der Komplexität genetischer Zusammenhänge und des Einflusses von Umweltfaktoren noch für die Bestimmung von Biomarkern für eine sichere klinische Diagnose oder von Progredienzmarkern für den Verlauf der Erkrankung reichen die 6 Patienten, die J. Parkinson beispielhaft beschrieb. In großen Kohorten, Registern und Programmen wird daher national und international unermüdlich nach Ursachen, Bio- und Progredienzmarkern geforscht.

\section{》) Die Parkinson-Erkrankung bleibt auch nach 200 Jahren eine klinische Diagnose}

Und dennoch hat sich an den Kernkriterien für die Diagnose wenig geändert. Vielmehr bildet der Zugang von J. Parkinson zu den Menschen, die er beschrieb, nämlich genaue Anamnese und exakte (longitudinale) Beobachtung, die Basis für die neuen klinischen Kriterien, die 2015 von einer Task Force der Movement Dis- orders Society veröffentlich wurden [3]. Die neuen Kriterien (siehe Beitrag von Zach et al. in diesem Heft) sollen das Vorgehen eines Experten festschreiben und einem Nichtexperten zugänglich machen [3]. Somit bleibt die Parkinson-Erkrankung auch nach 200 Jahren eine klinische Diagnose, auch wenn hilfreiche diagnostische Zusatzuntersuchungen (siehe Beitrag von Walter et al. in diesem Heft) die Differenzialdiagnose erleichtern.

Von J. Parkinson sehnlich erhofft, aber noch undenkbar, war lange Zeit eine Therapie der Erkrankung, die, so J. Parkinson schon 1817 „if employed before the disease had been too long established" sogar kurativ sein könnte.

Die mit dem Nobelpreis gekrönte Entdeckung von Dopamin und seinen Auswirkungen auf das Gehirn und damit die Parkinson-Erkrankung führte zur Entwicklung einer Vielzahl von v. a. dopaminergen, aber auch nichtdopaminergen medikamentösen Therapieansätzen, die neben den motorischen Symptomen in den letzten Jahren zunehmend auch die z. T. schon von J. Parkinson als den Patienten beeinträchtigend beschriebenen nichtmotorischen Symptome lindern (siehe Beitrag von Jost in diesem Heft bezüglich motorischer Symptome. Das umfangreiche Gebiet der Therapie nicht-motorischer Symptome wird in einer der folgenden Ausgaben des Nervenarztes in einer Übersichtsarbeit dargestellt werden). Der längere Erhalt der Alltagsaktivität der durch die individuellen, symptomorientierten Therapien ermöglicht wird, wird in den letzten Jahren zunehmend und erfolgreich durch nichtmedikamentöse Therapiestrategien konventioneller und interventioneller nen vom unteren Hirnstamm her aus 
Art (siehe Beitrag von Witt et al., in diesem Heft) unterstützt.

Somit ist ein Leben mit dem von J. Parkinson beschriebenen Übel („evil“) mit weitgehend zufriedenstellender Symptomkontrolle über längere Phasen möglich.

Allerdings kann trotz intensiver Forschung das Fortschreiten der Erkrankung bis heute weder gestoppt noch die Krankheit vermieden oder geheilt werden.

Hier könnten neue Therapieansätze, die sich genetische und pathomechanismenspezifische Ursachenforschung zunutze machen, neue Perspektiven bieten (siehe Artikel von Brockmann und Berg in diesem Heft). Um diese effektiv einzusetzen, ist ein tieferes Verständnis der Subgruppen der Erkrankung ebenso wichtig (siehe Artikel von Lill und Klein in diesem Heft) wie ein Verständnis des individuellen Verlaufs und die Bestimmung sinnvoller Endpunkte (siehe Artikel von Walter et al., in diesem Heft), die den Erfolg klinischer Studien messen können.

Und wie zur Zeit von J. Parkinson gilt es, den „Leidenden“ nicht aus dem Blick zu verlieren. Patienten und wissenschaftliche Gremien fordern zu Recht zunehmend, Lebensqualität in den Vordergrund der Behandlung zu stellen (siehe Artikel von Brockmann und Berg in diesem Heft). Dies muss mehr denn je Ziel aller die Betroffenen Begleitenden sein.

Die in diesem Heft veröffentlichten Artikel machen klar, dass aufbauend auf der von J. Parkinson verfassten faszinierend genauen und nachhaltigen Beschreibung des Krankheitsbildes der nach ihm benannten Erkrankung die Wissenschaft das Feld trotz noch vieler offenen Fragen substanziell weitergebracht hat. Ich bin überzeugt dass sich die Literatur jedes einzelnen Artikels sowohl für den Spezialisten wie auch für den Interessierten lohnt. Die Autoren haben speziell darauf geachtet, komplexe Sachverhalte so darzustellen, dass sie auch im klinischen Alltag strukturiert angewendet werden können.

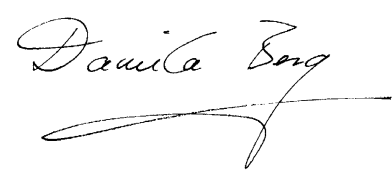

Prof. Dr. Daniela Berg

\section{Korrespondenzadresse}

\section{Prof. Dr. D. Berg}

Klinik für Neurologie, Christian-AlbrechtsUniversität zu Kiel

Arnold-Heller-Str. 3, 24105 Kiel, Deutschland

Daniela.Berg@uksh.de

Interessenkonflikt. D. Berg gibt an, dass kein Interessenkonflikt besteht.

\section{Literatur}

1. Parkinson J (1817) An Essay on the shaking palsy. Writtingham and Rowland, London

2. Braak H et al (2002) Staging of the intracerebral inclusion body pathology associated with idiopathic Parkinson's disease (preclinical and clinical stages). J Neurol 249(Suppl 3:III):1-5

3. Postuma RB et al (2015) MDS clinical diagnostic criteria for Parkinson's disease. Mov Disord 30(12):1591-1601
Theo und Friedl Schöller-Preis für Alternsforschung 2017

Schwerpunkt: Altersmedizin

Mit dem Theo und Friedl Schöller-Preis werden herausragende, bereits fertiggestellte wissenschaftliche Arbeiten aus den letzten beiden Jahren ausgezeichnet, die sich besonders mit den Belangen multimorbider, hochaltriger Menschen befassen und somit neue Aspekte in Forschung und Lehre eröffnen.

Bewerben können sich im deutschsprachigen Raum tätige Mediziner, die im Bereich der Altersmedizin forschen, und Vertreter angrenzender Wissenschaften aus den Bereichen der Lebens-, Natur- und Ingenieurwissenschaften sowie aus Geistes-, Pflege-, Sozialwissenschaften und der Gesundheitsökonomie.

Das Preisgeld beträgt $€ \mathbf{2 0 . 0 0 0 , -}$ Ausschreibungsende: 16.06.2017

Alle Vorschläge sind mit einem eigenständigen Antrag, einer Darstellung der wissenschaftlichen Arbeit, der Kurzbiographie des Bewerbers sowie einer Empfehlung einzureichen. Jeder Bewerber oder jede Arbeitsgruppe darf im Rahmen der Ausschreibung nur eine Arbeit oder ein Forschungsprojekt vorlegen. Einzureichen ist ferner eine Versicherung, dass die Arbeit oder das Projekt nicht für eine andere Ausschreibung eingereicht oder vorgesehen ist oder von anderer Seite bereits ausgezeichnet wurde. Die Arbeit soll zumindest in der Zusammenfassung in deutscher Sprache vorliegen. Der für die Bewerbung notwendige Leitfaden ist auf der unten genannten Website abzurufen.

Die Bewerbung ist in elektronischer Form unter folgender Adresse einzureichen:

Zentrum für Altersmedizin im Klinikum Nürnberg

z.Hd. Frau Elke Kaufmann

Prof.-Ernst-Nathan-Straße 1

90419 Nürnberg

Tel: 0911-398-3917

E-Mail: alterszentrum@klinikum-nuernberg.de

www.altersmedizin-nuernberg.de

Das Zentrum für Altersmedizin steht für Rückfragen gerne zur Verfügung. 